\title{
Irrelevant speech, articulatory suppression, and phonological similarity: A test of the phonological loop model and the feature model
}

\author{
J. RICHARD HANLEY and EIRINI BAKOPOULOU \\ University of Essex, Colchester, England
}

\begin{abstract}
Two experiments tested competing predictions about the nature of the irrelevant speech effect that were derived from Neath's (2000) feature model and from Salamé and Baddeley's (1982) phonological loop model. The first experiment examined the combined effects of irrelevant speech and articulatory suppression when target items were presented auditorily. Contrary to the suggestions of Neath, but consistent with the phonological loop model, the effects of articulatory suppression and irrelevant speech were additive even when the irrelevant speech was presented during the retention interval. The second experiment examined the combined effects of irrelevant speech and phonological similarity when target items were presented visually. Consistent with the phonological loop model, the effects of phonological similarity and irrelevant speech were additive when participants were specifically instructed to use articulatory/phonological rehearsal to remember the list items. The results therefore contradicted Neath's claim that irrelevant speech abolishes the phonological similarity effect when list items are presented visually. However, the effect of phonological similarity was abolished in the irrelevant speech conditions when no instructions were given concerning rehearsal. It is argued that the phonological similarity effect disappears in some experiments because participants sometimes employ a semantic rehearsal strategy, consistent with the views of Salamé and Baddeley (1986).
\end{abstract}

Immediate serial recall of verbal material is reduced significantly if participants are exposed to irrelevant speech while studying the list items. This effect occurs despite the fact that participants are told they should ignore the irrelevant material. The irrelevant speech effect was first reported by Colle and Welsh (1976) with visual presentation of list items and has since been demonstrated in a large number of published studies (e.g., Ellermeier \& Zimmer, 1997; Jones, 1994; Jones \& Macken, 1995; Jones, Madden, \& Miles, 1992; Salamé \& Baddeley, 1982; Surprenant, LeCompte, \& Neath, 2000). The irrelevant speech effect also occurs when the list items are presented auditorily (e.g., Hanley \& Broadbent, 1987; Neath, Surprenant, \& LeCompte, 1998).

Recently, Neath (2000) has put forward a computational model of serial recall, known as the feature model, that attempts to explain why irrelevant speech reduces recall performance. One of Neath's main goals was to provide a plausible explanation of the way in which irrelevant speech interacts with three other variables that are also known to affect serial recall: articulatory suppression, phonological similarity, and word length. Neath's model differs in a number of ways from Salamé and Baddeley's (1982) ac-

Correspondence should be addressed to J. R. Hanley, Department of Psychology, University of Essex, Wivenhoe Park, Colchester CO4 3SQ, England (e-mail: rhanley@essex.ac.uk). count of the interrelationship between these variables, which is based on the phonological loop component of working memory (e.g., Baddeley, 1986). The present paper tests the competing predictions that these two theories make about the relationship between irrelevant speech and articulatory suppression (Experiment 1) and about the relationship between irrelevant speech and phonological similarity (Experiment 2).

\section{Phonological Loop Account of the Irrelevant Speech Effect}

According to Salamé \& Baddeley (1982), the effects of irrelevant speech are explicable in terms of the operation of the phonological loop (e.g., Baddeley, 1986, 1990, 1992). Salamé and Baddeley (1982) argued that irrelevant speech interferes with the temporary storage of verbal material within a phonological input store of limited capacity. The phonological store, together with the process of articulatory rehearsal, constitute what is known as the phonological loop. Spoken material has obligatory access to the phonological store, whereas visually presented information will enter the phonological store if it is articulated. Figure 1 provides a flow diagram that illustrates some of the functions of the main components of the phonological loop model. Consistent with this account, Salamé and Baddeley (1982) showed that when the list items were presented visually, the effects of irrelevant speech were removed by articulatory suppression. Similar findings were 


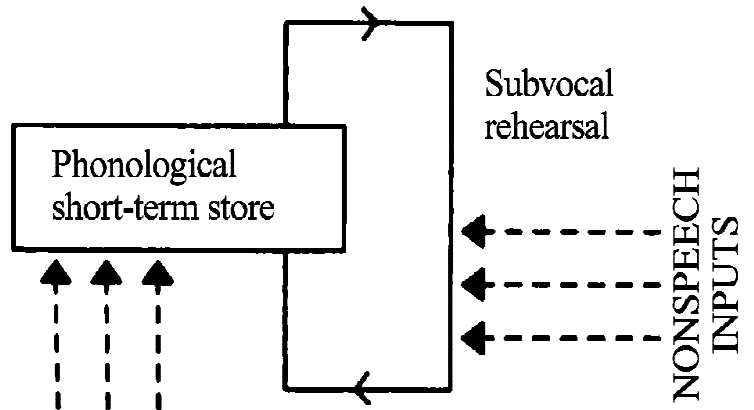

SPEECH INPUTS

Figure 1. A representation of the phonological loop model taken from Gathercole and Baddeley (1993). Speech inputs (e.g., irrelevant speech and auditorily presented list items) gain automatic entry to the phonological store. Nonspeech inputs (e.g., visually presented list items) require articulatory rehearsal in order to access the phonological store and are therefore cut off from the phonological store by articulatory suppression. Irrelevant speech will interfere with the representations of list items if and only if they are being held within the phonological store. The form in which visually presented words or letters are stored under suppression is not specified by the model.

reported by Hanley (1997). Salamé and Baddeley (1982) claimed the reason for this interaction is that concurrent articulation isolates visually presented material from the phonological store. Although performance will be lower as a consequence, it will not be further reduced by exposure to irrelevant speech.

It follows from the phonological loop model that if the target material is presented auditorily, then irrelevant speech should reduce serial recall performance under articulatory suppression. This is because the auditorily presented list items will enter the phonological store automatically. As a consequence, list items will be subject to interference from irrelevant speech even though concurrent articulation is taking place. This prediction was supported by Hanley and Broadbent (1987). They showed additive effects of irrelevant speech and articulatory suppression on the recall of auditorily presented lists of items in two out of three experiments even when articulatory suppression continued during presentation and recall. We investigate this issue further in Experiment 1.

The phonological loop model also provides an account of the relationship between the effects of irrelevant speech and the effects of phonological similarity (Baddeley, 1966, 1968; Conrad \& Hull, 1964) on serial recall. It is claimed (e.g., Baddeley, Lewis, \& Vallar, 1984) that the representations of phonologically similar items cause interference to one another within the phonological store. Consistent with this claim, a number of studies have shown that with visual presentation of list items, the effects of phonological similarity are also abolished by articulatory suppression (e.g., Baddeley et al., 1984; Longoni, Richardson, \& Aiello, 1993; Murray, 1967, 1968). Because both the irrelevant speech effect and the phonological similarity effect reflect the operation of the phonological store, it follows from the phonologicalloop model that the effects of phonological similarity and irrelevant speech should be additive. In other words, phonologically similar items should be recalled worse than phonologically different items even in the presence of irrelevant speech. Although this prediction has been supported in some studies (Jones \& Macken, 1995; ${ }^{1}$ Larsen, Baddeley, $\&$ Andrade, 2000; Salamé \& Baddeley, 1986), the evidence is mixed. Neither Colle and Welsh (1976) nor Surprenant, Neath, and LeCompte (1999) observed a phonological similarity effect in the presence of irrelevant speech. We investigate this issue further in Experiment 2.

\section{Feature Model Account of the Irrelevant Speech Effect}

Neath's (2000) alternative model of the irrelevant speech effect is based on Nairne's (1990) feature model. The feature model assumes that each item from a list is represented in memory as a discrete set of features that are set to either 1 or -1 . Every item is represented in both primary memory and secondary memory. Although secondary memory traces are considered to be intact, traces in primary memory are subject to interference from later items. The presentation of a new item that shares a feature value with one or more items from earlier in the list will have the effect of altering the value of that feature from 1 or -1 to zero in the memory traces of all the previously presented items. This renders the feature useless for the purposes of recalling the earlier items. At recall, each trace in primary memory is used to access the most similar trace in secondary memory with the trace that has greatest feature overlap with the trace in primary memory becoming selected for recall. As features become lost from the trace of the item in primary memory, so the probability increases that an incorrect item will be recalled.

The features that represent list items are either modalityindependent or modality-dependent features. The former are activated regardless of modality of presentation, whereas different modality-dependent features are activated when an item is presented visually from those that are activated when it is presented auditorily. It is assumed that there are more modality-dependent features associated with the auditory modality than with the visual modality (hence the modality effect in serial recall).

\section{The Effects of Irrelevant Speech and Articulatory Suppression}

According to Neath (2000), irrelevant speech and articulatory suppression reduce recall in broadly similar ways. The word that is repeated by the participant during articulatory suppression causes additional interference to previously presented list items. Some of the modality-independent features in primary memory are replaced by features of the word that is articulated, a process referred to as feature adoption. To simulate this effect, half of the modalityindependent features in each trace are set to a constant value $(+1)$. Feature adoption is also the cause of the interference that is brought about by irrelevant speech. Some of the features of the words in the irrelevant speech stream replace features of the traces of the target items in 
primary memory. This process is simulated by replacing half of the modality-independent features in each trace by a random combination of $+1 \mathrm{~s}$ and $-1 \mathrm{~s} .^{2}$

On the basis of the claim that irrelevant speech and articulatory suppression exert similar effects on traces in primary memory, Neath (2000) argued that there will be no extra interference from irrelevant speech under conditions of articulatory suppression. Here the predictions of the feature model are in conflict with the predictions of the phonologicalloop model. The phonologicalloop model and the feature model both predict that there will be no effect of irrelevant speech under conditions of articulatory suppression when list items are presented visually. With auditory presentation of list items, however, the phonological loop model predicts additive effects of articulatory suppression and irrelevant speech, whereas the feature model predicts a reduced effect of irrelevant speech when articulation is suppressed. These rival predictions were investigated in Experiment 1.

\section{The Effects of Irrelevant Speech and Phonological Similarity}

Under normal conditions in Neath's (2000) simulations, a random sample of $50 \%$ of the features in any two traces will be set to the same value. The effects of phonological similarity are simulated by setting a consistent set of features to the same value in phonologically related items. This means that there will be a higher proportion of shared features in the traces of list items than would be the case in phonologically different lists. By the time of recall, therefore, virtually all of these feature values will have been turned to zeros in the primary memory traces, and performance will be relatively poor as a consequence. With visual presentation of list items, the feature model predicts that there will be no effect of phonological similarity under conditions of irrelevant speech. This is because recall of visually presented material depends heavily on modalityindependent features (there are limited modality-dependent features associated with the visual modality). However, irrelevant speech has already interfered with the representations of modality-independentfeatures, and phonological similarity will produce no further damage to them.

Here again, the predictions of the feature model and the phonological loop model are in conflict. The phonological loop model predicts that irrelevant speech and phonological similarity should be additive for visually presented list items. As we saw earlier, previous research on this issue has produced mixed results, with only some of the published studies demonstrating additive effects of phonological similarity and irrelevant speech. In Experiment 2, we investigated possible reasons for these inconsistencies.

\section{EXPERIMENT 1}

Neath's (2000) feature model predicts that there should be no effect of irrelevant speech under articulatory suppression when list items are presented auditorily (see the right panel of Simulation 6, Neath, 2000, p. 418). The only previously published investigation of this issue was pro- vided by Hanley and Broadbent (1987). In their first experiment, they found that articulatory suppression removed the irrelevant speech effect. However, there were some reasons why the sensitivity of this experiment may have been limited. First, different participants were used in the speech and quiet conditions. Second, list length was long (nine items per list), and it has been claimed that participants might stop using the phonological loop altogether when conditions are very difficult (Baddeley, 2000). In their final experiment, Hanley and Broadbent employed a within-subjects experiment and attempted to make the task easier by reducing list length to six items per list. Under these circumstances, there were additive effects of irrelevant speech and articulatory suppression, consistent with the phonological loop model. Neath suggested that these results might have come about because the irrelevant speech masked the perception of the auditorily presented target items. This masking would have caused an additional detrimental effect on recall under conditions of articulatory suppression. Hanley and Broadbent (p. 291) argued against such an explanation by demonstrating unimpaired perception of auditorily presented target items when accompanied by irrelevant speech. Experiment 1 sought to investigate this issue further by presenting the irrelevant speech during the retention interval between presentation and recall instead of during input.

In Experiment 1, therefore, a technique used previously by Miles, Jones, and Madden (1991) was employed. Using visual presentation of target material, Miles et al. showed that the effects of irrelevant speech were as strong when it was presented during the retention interval as when it was presented at the same time as the list items. In Experiment 1, we used Miles et al.'s procedure with auditory presentation of list items. Half of the participants were exposed to irrelevant speech during the retention interval and half were exposed to irrelevant speech during list presentation. If some of the effects of irrelevant speech on auditorily presented list items come about because of perceptual masking at encoding, then these effects should be significantly reduced if the irrelevant speech is presented during the retention interval only. If there is an effect of irrelevant speech under articulatory suppression simply because the irrelevant speech masks the target items, then there should be additive effects of irrelevant speech and articulatory suppression when irrelevant speech is presented during list presentation. When irrelevant speech is presented during the retention interval, there should be an interaction between irrelevant speech and articulatory suppression such that there is no effect of irrelevant speech in the articulatory suppression conditions. The phonological loop model, on the other hand, predicts that the irrelevant speech should exert similar effects on recall when articulation is suppressed regardless of whether the speech occurs during list presentation or the retention interval.

\section{Method}

Participants. Forty students at Essex University volunteered to take part in the experiment and were tested individually. Twenty participants were assigned at random to the two experimental groups. 
Design. The experiment employed a mixed factorial design. Location of irrelevant speech (during list presentation or during the retention interval) was manipulated between subjects. Irrelevant speech (present vs. absent) and articulatory suppression (present vs. absent) were manipulated within subjects. Each participant therefore took part in four different conditions: control, articulatory suppression alone, irrelevant speech alone, and articulatory suppression plus irrelevant speech. The order in which participants performed these conditions was counterbalanced by the use of a Latin square.

Materials. All lists contained six target items. The lists comprised randomly ordered sequences of the digits 1-9 sampled with replacement. A total of 60 such lists were prepared, with 15 lists in each of the four conditions. The first three lists in each block were treated as practice lists. Materials were presented by an Apple Macintosh using the Quicktime application. The Final Cut-Pro application was used to edit the tapes.

Procedure. Each participant wore stereo headphones for the duration of the experiment. A 1-sec tone presented in the right headphone alerted participants to the start of each list. One second after the tone, they heard the target items through the right headphone spoken by a female voice at a rate of one digit per second. Each participant listened to 60 lists of items, separated into four blocks containing 15 lists each with a break between blocks while the new set of instructions was presented. For two blocks, once in the presence of irrelevant speech and once without, the participant had to repeat the word and at a rate of approximately two repetitions per second during presentation, retention interval, and recall of the target stimuli. Participants' articulation was monitored to ensure that a steady rate was maintained throughout the experiment.

In all lists, there was a retention interval of $10 \mathrm{sec}$ (the same retention interval as that used by Miles et al., 1991) between presentation of the final item and recall of the target items. Participants were told that they should try to remember the numbers by repeating them silently to themselves during the retention interval. A further tone sounded to mark the end of the retention interval. This was the signal for participants to commence recall of the list items. They were told to write the list items down in the order in which they had heard them, and that they were not allowed to change any earlier items once they had written down a subsequent item. Their recall sheet contained six boxes for each list corresponding to the six serial po- sitions. They were allowed $12 \mathrm{sec}$ to recall the digits before a further tone sounded to tell them to stop recall. There was then a gap of $5 \mathrm{sec}$ before the tone sounded again to signal the start of the next list.

Irrelevant speech was presented through the left headphone spoken by a male voice. Irrelevant speech comprised letters of the alphabet being read out in a random order at the rate of approximately one and a half letters per second. All 60 lists were associated with a different 6-sec segment of irrelevant speech. For half of the participants, the irrelevant speech occurred during list presentation. For these participants, the irrelevant speech started exactly $1 \mathrm{sec}$ after presentation of the first digit and continued until $1 \mathrm{sec}$ after the final list item had been presented. The other half of the participants heard the irrelevant speech during the last $6 \mathrm{sec}$ of the retention interval. For each list, exactly the same token of irrelevant speech was used regardless of whether it occurred during presentation or during the retention interval. This ensured that the "dose" (Bridges \& Jones, 1996) of the irrelevant speech was identical regardless of whether it was presented at encoding or during the retention interval.

\section{Results}

A significance level of .05 (two-tailed) was established for all statistical tests reported in this paper. Overall mean performance in Experiment 1 is summarized in Figure 2. A three-way analysis of variance was performed on the number of items correctly recalled in serial order. This revealed a significant main effect of irrelevant speech $\left[F(1,28)=39.5, M S_{\mathrm{e}}=31.9\right]$, a significant main effect of articulatory suppression $\left[F(1,38)=277.0, M S_{\mathrm{e}}=56.1\right]$, but no effect of location of the irrelevant speech $(F<1)$. The interaction between articulatory suppression and irrelevant speech was significant $\left[F(1,38)=8.1, M S_{\mathrm{e}}=\right.$ 31.4]. Neither of the remaining two-way interactions approached significance. The three-way interaction also failed to reach significance $(F<1)$.

The significant interaction between articulatory suppression and irrelevant speech was further investigated by tests of simple main effects. These revealed significant ef-

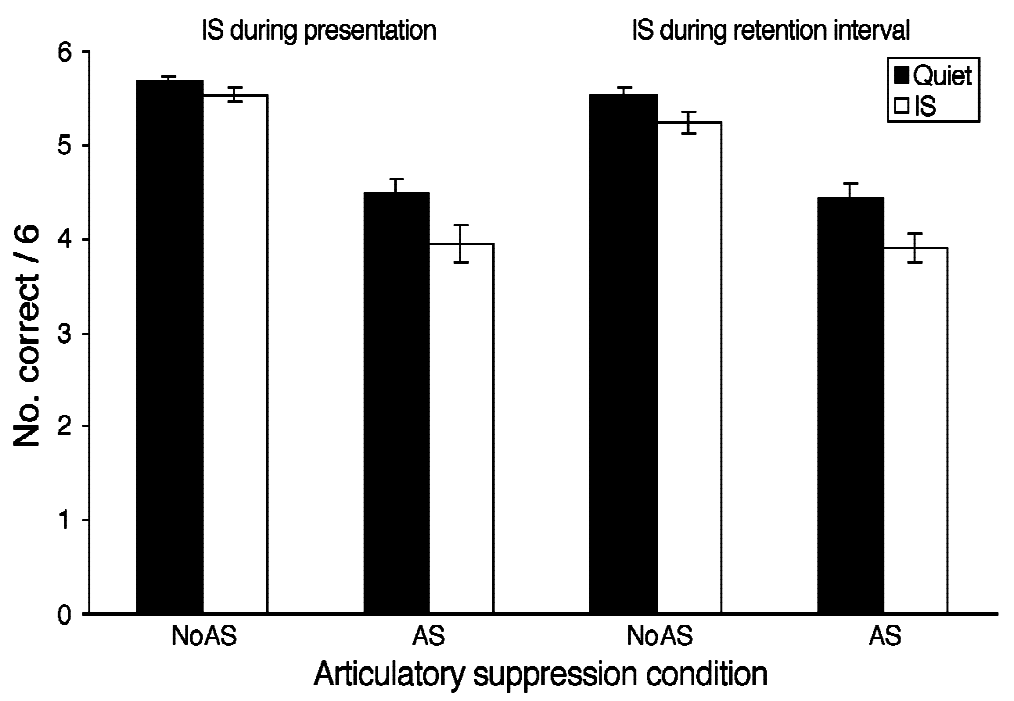

Figure 2. Mean number (plus standard error bars) of phonologically distinct and similar items correctly recalled per list $(\max =6)$ with articulatory suppression (AS) and without articulatory suppression (NoAS) in the presence and absence of irrelevant speech (IS) in Experiment 1. 
fects of irrelevant speech both with articulatory suppression $\left[F(1,76)=41.8, M S_{\mathrm{e}}=31.6\right]$ and without articulatory suppression $\left[F(1,76)=6.1, M S_{\mathrm{e}}=31.6\right]$. The effects of articulatory suppression were significant both with irrelevant speech $\left[F(1,76)=225.9, M S_{\mathrm{e}}=43.7\right]$ and without irrelevant speech $\left[F(1,76)=135.3, M S_{\mathrm{e}}=43.7\right]$. The interaction between irrelevant speech and articulatory suppression seems to have occurred because the effects of irrelevant speech are somewhat larger with rather than without articulatory suppression (Figure 2). The most likely explanation is that the effects of irrelevant speech are being reduced slightly in the no-suppression condition as a result of a ceiling effect.

\section{Discussion}

The results of this experiment have replicated those of the second and third experiments by Hanley and Broadbent (1987) in showing significant effects of irrelevant speech on auditorily presented items under conditions of articulatory suppression, consistent with Salamé and Baddeley's (1982) phonologicalloop model. Because auditorily presented items enter the phonological store automatically, they will be subject to interference from irrelevant speech even under conditions in which participants perform articulatory suppression. The results are contrary to the predictions of Neath's (2000) feature model. Neath argued that articulatory suppression and irrelevant speech both interfere with the representations of modalityindependent features in primary memory traces. Once articulatory suppression has affected these memory traces, irrelevant speech should cause them no further damage.

The findings reported here extend those of Hanley and Broadbent (1987) by showing significant effects of irrelevant speech under conditions of articulatory suppression even when the irrelevant speech was presented during the retention interval (Figure 2). Neath (2000) suggested that the effects of irrelevant speech under articulatory suppression that Hanley and Broadbent reported might have come about because the irrelevant speech masked the perception of the auditorily presented target words. If this suggestion were true, one would have expected that the effects of irrelevant speech should have been greatly reduced when the irrelevant speech was presented during the retention interval. This is because there can be no perceptual masking when the irrelevant speech starts several seconds after the target items have been presented. The absence of any relationship between the location of the irrelevant speech and the strength of the irrelevant speech effect (Figure 2) shows that this is not the case. If Neath's suggestion were true, there should also have been additive effects of irrelevant speech and articulatory suppression when the irrelevant speech occurred at encoding, but an interaction between articulatory suppression and irrelevant speech when the irrelevant speech occurred during the retention interval. The complete absence of any threeway interaction shows that this is not the case. The strength of the irrelevant speech effect under articulatory suppression is just as strong when the irrelevant speech occurs in the retention interval as when it occurs during list presen- tation (Figure 2). The results therefore further confirm one of the key predictions about the irrelevant speech effect made by Salamé and Baddeley's (1982) phonological loop model.

It is well known that the presentation of a single item, or "suffix," immediately after the final item in a list can significantly reduce serial recall of auditorily presented lists (e.g., Crowder, 1967). According to some (e.g., Crowder \& Morton, 1969), the suffix interferes with the precategorical representation of the final list item within auditory sensory memory. Is it conceivable, therefore, that the effect of irrelevant speech in Experiment 1 came about because the speech functioned as a suffix? If so, the feature model could accommodate the results from Experiment 1. This is because the feature model (see Surprenant et al., 2000) assumes that a suffix interferes with the modality-dependent features of the final list item (whereas irrelevant speech and articulatory suppression interfere with modalityindependent features). However, it seems unlikely that the effects of irrelevant speech when presented during the retention interval acted simply as a suffix. First, Crowder (1978) reported that a suffix had little effect if presented more than $2 \mathrm{sec}$ after the final list item, but in Experiment 1 there was a delay of $6 \mathrm{sec}$ between the presentation of the final list item and the start of the irrelevant speech. Second, there is no evidence that the effects of a suffix can persist over a retention interval as long as that used in Experiment $1(10 \mathrm{sec})$. For example, Watkins and Todres (1980) showed that unless the participants were asked to perform a secondary task during the retention interval, a suffix exerted no effect on serial recall if there was a delay of $12 \mathrm{sec}$ between the end of the list and recall. Finally, Morton, Crowder, and Prussin (1971) showed that the effects of a suffix were substantially reduced if the targets were presented to one ear and the suffix to the other. Morton et al. also reported that a pure tone did not produce a suffix effect. Irrelevant speech effects, on the other hand, are produced by sequences of changing-state auditory tones (Jones \& Macken, 1993). It would therefore be possible to investigate this issue further by comparing the effects of irrelevant tones and irrelevant words when presented during the retention interval following auditory presentation of list items under conditions of articulatory suppression.

\section{EXPERIMENT 2}

In Experiment 2, we investigated competing accounts of the combined effects of irrelevant speech and phonological similarity on serial recall. As was pointed out earlier in the paper, some of the available evidence (Jones \& Macken, 1995; Larsen et al., 2000; Salamé \& Baddeley, 1986) is consistent with the phonological loop model because it demonstrates a detrimental effect of phonological similarity on the recall of visually presented list items when participants are exposed to irrelevant speech. In other studies employing visual presentation of target materials (Colle \& Welsh, 1976; Surprenant et al., 1999), however, there was no effect of phonological similarity in the presence of irrelevant speech. These two studies are therefore 
consistent with the feature model (Neath, 2000), which predicts that under these circumstances the phonological similarity effect will be abolished by irrelevant speech.

Salamé and Baddeley (1986) offered an explanation for why phonological similarity effects sometimes do not occur in the presence of irrelevant speech. In their own study, they varied list length from 5 to 8 items per list. They found clear additive effects at List Lengths 5 and 6. At List Lengths 7 and 8, the interaction between phonological similarity and irrelevant speech approached significance, and at List Length 8 there was no main effect of phonologicalsimilarity. Salamé and Baddeley (1986) suggested that at short list lengths, their participants adopted an articulatory/phonological rehearsal strategy that made use of the phonologicalloop. Salamé and Baddeley (1986) argued that when lists contain large numbers of phonologically similar items, however, phonological coding is likely to be difficult and will be abandoned by many participants in favor of a visual or semantic strategy. In support of this account, they reported that high-performing participants showed additive effects of phonological similarity and irrelevant speech even with a list length of eight items. They suggested that these individuals had greater phonological loop capacity and were able to maintain a phonological coding strategy throughout the experiment.

Colle and Welsh (1976) and Surprenant et al. (1999) both used relatively long lists (eight items per sequence). Consequently, Salamé and Baddeley's (1986) account can explain the absence of an effect of phonological similarity in the presence of irrelevant speech in these studies also by assuming that many participants abandoned a phonologicalcoding strategy. Salamé and Baddeley's (1986) account is also consistent with the results of Larsen et al. (2000), who showed additive effects of irrelevant speech and phonological similarity in two experiments that employed relatively short sequences (six items per list).

Nevertheless, there is no direct evidence to support Salamé and Baddeley's (1986) claim that significant effects of phonological similarity are dependent on the use of a phonological coding strategy. Nor is there direct evidence that a semantic strategy will abolish the effects of phonological similarity. In Experiment 2, therefore, we sought to investigate these issues further by giving some of the participants explicit instructions on how they should encode the target items. One group was told to use a phonological rehearsal strategy. We assumed that this group would use the phonological loop to rehearse the target items. On the basis of Salamé and Baddeley's (1986) account, it was therefore predicted that this group would show additive effects of irrelevant speech and phonological similarity. Another group was instructed to use a semantic strategy, and it was assumed that they would not use the phonological loop. It was therefore predicted that this group would show no effect of irrelevant speech or phonologicalsimilarity. A third group was given no instructions (standard procedure in serial recall experiments). We used list lengths of seven items per sequence because this is the length at which Salamé and Baddeley's (1986) results suggested that some participants might abandon a phonological strategy in the presence of irrelevant speech when items are phonologically similar. It was predicted that the performance of this group would resemble that of the participants in Colle and Welsh's (1976) and Surprenant et al.'s (1999) studies in showing effects of phonological similarity only in quiet conditions.

\section{Method}

Participants. Fifty-four students at Essex University took part in the experiment and were tested individually. They were either paid $£ 3$ or received a course credit for taking part. There were 16 participants in the phonological group, 16 participants in the semantic group, and 22 participants in the control group.

Materials. All lists of target items contained seven randomly ordered items, sampled without replacement, from either a set of phonologically similar letters (B, C, D, G, P, T, V) or a set of phonologically different letters (H, J, R S, L, Y, Z). The irrelevant speech consisted of a tape recording of a passage from Bleak House by Charles Dickens read by a native English speaker.

Design and Procedure. Irrelevant speech (present vs. absent) and phonological similarity (similar vs. different) were both manipulated within subjects. Each participant received a total of 48 lists divided into two blocks of 24 lists each. Participants were exposed to irrelevant speech during one block of lists, and one block was presented in silence. Half of the participants heard irrelevant speech during the first block of lists, and half of the participants heard irrelevant speech during the second block of lists. Irrelevant speech was presented through a loudspeaker during list presentation and during recall of list items. Participants were told to ignore the irrelevant speech. The first four lists during each block were deemed practice lists. Half of the critical and half of the practice lists in each block contained phonologically similar letters, and half contained phonologically different letters. The order of the phonologically similar and different lists within each block was randomized.

Target items were presented one at a time in uppercase on the screen of an Apple Macintosh computer. At the start of each list, the word ready appeared for $0.75 \mathrm{sec}$ on the computer screen. The screen then went blank for $0.5 \mathrm{sec}$ before the first target letter appeared in 48-point Geneva font. Each letter remained on the screen for $1.0 \mathrm{sec}$ with an interstimulus interval of $0.5 \mathrm{sec}$. After the final letter disappeared from the screen, a tone sounded for $0.25 \mathrm{sec}$. Participants were then allowed $12 \mathrm{sec}$ to attempt to recall the list items. When the $12 \mathrm{sec}$ was over, the tone sounded and participants were asked to look at the screen once again for the start of the next list. Recall instructions were the same as those used in Experiment 1.

Before the experiment began, the participants were given one of three different sets of instructions. The participants in the semantic group were told to try and remember the letters by turning each one into a word that started with that letter. They were then told to attempt to create a meaningful sentence from the words that they had generated, maintaining the order in which the letters had been presented. They could then use the sentence to help them recall the order of the letters at test. The phonological group was told to remember the letters by repeating them subvocally until they had to be recalled. The control group was given no instructions as to how to remember the items. At the end of the experiment, all participants were asked to describe the strategies that they had used to remember the list items. All of the participants in the phonological group indicated that they had used a phonological strategy throughout the experiment. Sixty-nine percent of the participants in the semantic group reported consistent use of the strategy they had been asked to employ. The strategies employed in the control group are discussed below.

\section{Results}

Overall mean performance in Experiment 2 is summarized in Figure 3. 
An analysis of performance in the phonological group revealed a significant main effect of irrelevant speech $\left[F(1,15)=13.4, M S_{\mathrm{e}}=435.8\right]$ and a significant main effect of phonological similarity $\left[F(1,15)=483.5, M S_{\mathrm{e}}=50.4\right]$. The interaction between irrelevant speech and phonological similarity was not significant $\left[F(1,15)=1.3, M S_{\mathrm{e}}=\right.$ 37.6]. Planned comparisons (tests of simple main effects) revealed significant effects of phonological similarity in quietconditions $\left[F(1,15)=47.2, M S_{\mathrm{e}}=33.5\right]$ and in the presence of irrelevant speech $\left[F(1,15)=16.6, M S_{\mathrm{e}}=54.5\right]$.

An analysis of performance in the semantic group revealed no significant main effect of irrelevant speech $\left[F(1,15)=4.2, M S_{\mathrm{e}}=37.4, p=.06\right]$ or of phonological similarity $(F<1)$. The interaction between irrelevant speech and phonological similarity was not significant $(F<1)$. Planned comparisons (tests of simple main effects) revealed no significant effect of phonological similarity in quiet conditions or in the presence of irrelevant speech (both $F \mathrm{~s}<1$ ).

An analysis of performance in the control group revealed a significant main effect of irrelevant speech $\left[F(1,21)=4.3, M S_{\mathrm{e}}=62.1\right]$ and a significant main effect of phonological similarity $\left[F(1,21)=4.4, M S_{\mathrm{e}}=114.3\right]$. There was also a significant interaction between irrelevant speech and phonological similarity $\left[F(1,21)=4.8, M S_{\mathrm{e}}=\right.$ 22.0]. Planned comparisons (tests of simple main effects) revealed significant effects of phonological similarity in quiet conditions $\left[F(1,21)=6.4, M S_{\mathrm{e}}=82.7\right]$, but no significant effect of phonological similarity in the presence of irrelevant speech $\left[F(1,21)=1.4, M S_{\mathrm{e}}=53.6\right]$.

\section{Discussion}

The results of this experiment revealed strikingly different patterns of performance in the three groups of participants. When no encoding instructions were given, there was a significant effect of phonological similarity in quiet conditions but not in the presence of irrelevant speech. When participants were given phonological coding instructions, there were significant effects of phonological similarity even in the presence of irrelevant speech. With semantic instructions, there was no effect of phonological similarity even in quiet conditions.

The fact that there were additive effects of phonological similarity and irrelevant speech when participants were told to use articulatory/phonological rehearsal provides strong support for the phonological loop model (Salamé \& Baddeley, 1982). According to this model, visually presented material will enter the phonological store when it is articulated even in the presence of irrelevant speech. Once they have entered the phonological store, phonologically similar items will be more difficult to maintain than phonologically different items. Both types of item will also suffer interference from the irrelevant speech. Consequently irrelevant speech and phonological similarity should make separate contributions to reducing serial recall performance. Conversely, the feature model (Neath, 2000) predicts that irrelevant speech should abolish the phonological similarity effect with visual presentation of targets. The fact that there was a phonological similarity effect in the presence of irrelevant speech is therefore inconsistent with the feature model.

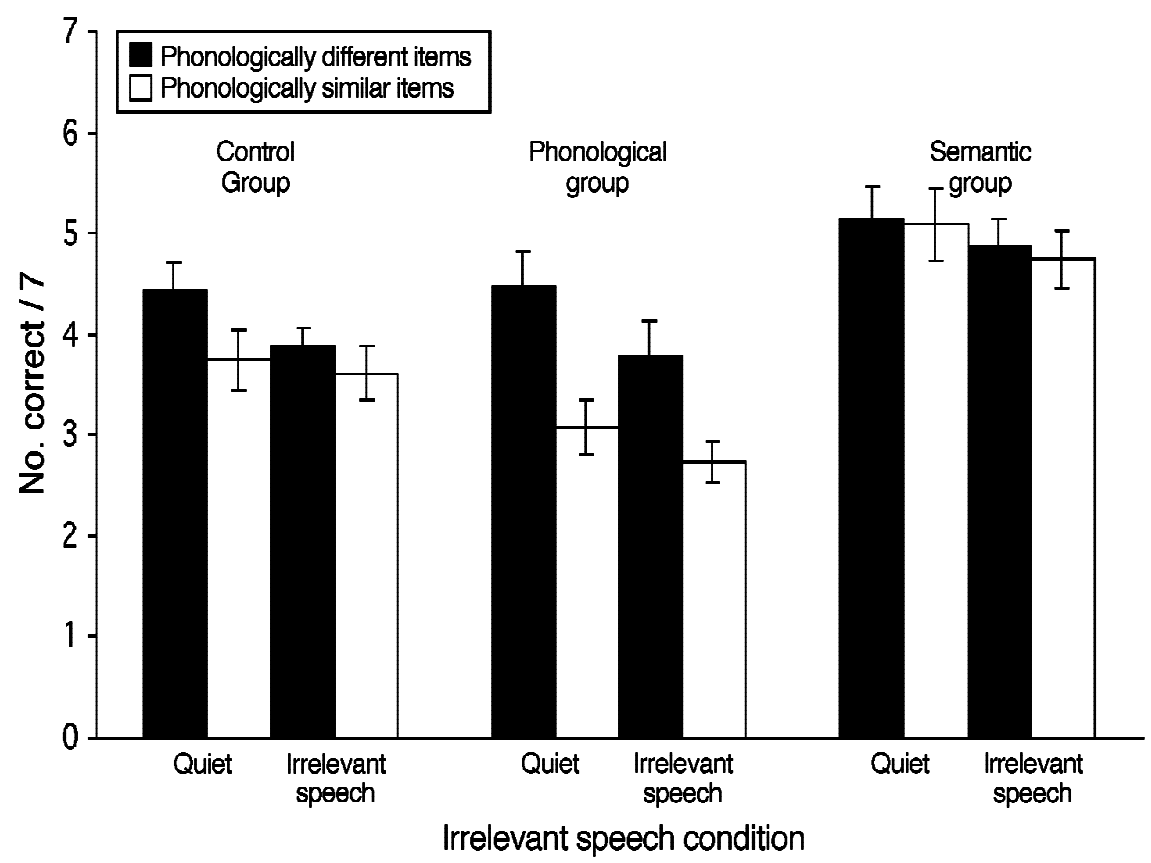

Figure 3. Mean number $( \pm S E)$ of phonologically different and phonologically similar items correctly recalled per list $(\max =7)$ by the three experimental groups in quiet conditions and in the presence of irrelevant speech in Experiment 2. 
The semantic group showed no effect of phonological similarity and a small, nonsignificant effect of irrelevant speech, with the majority of participants reporting that they had successfully used the semantic strategy that they had been asked to employ. Such an outcome shows that when instructed, many individuals can readily adopt alternative strategies that counteract the effects of variables that have long been known to interfere with serial recall performance. The performance of the semantic group in Experiment 2 therefore confirms the claim (Baddeley, 2000; Salamé \& Baddeley, 1986) that the effects of phonological similarity will disappear if participants switch to a semantic strategy as list length increases.

The finding that irrelevant speech removed the phonological similarity effect when participants were given no encoding instructions is similar to the results of Colle and Welsh (1976) and Surprenant et al. (1999). Because this result is quite different from that observed in the phonological group, it must be concluded that at least some of the participants in the control group were not using articulatory/phonological rehearsal strategies. Further support for this conclusion emerged when the participants in the control group were asked to outline the strategies they had used to remember the items. Whereas all of the participants in the phonological group reported using a phonological strategy, a wide range of different strategies was described by the control group. Six participants (27\%) in the control group reported that they consistently used an articulatory/phonological strategy. An analysis of the performance of these 6 participants revealed a significant effect of phonological similarity both in the presence $\left[F(1,5)=10.7, M S_{\mathrm{e}}=62.9\right]$ and absence $\left[F(1,5)=7.1, M S_{\mathrm{e}}=\right.$ 62.9] of irrelevant speech. This suggests that these individuals were indeed using phonological coding. Four participants $(18 \%)$ reported consistent use of a semantic strategy that involved converting the letters into words and making sentences or phrases from the words. These 4 participants showed no evidence whatsoever of a phonological similarity effect, performing slightly, though nonsignificantly, better overall on the phonologically similar items. Fourteen percent reported using a mixture of phonological and semantic strategies on different lists, with semantic strategies being used more frequently on the phonologically similar items. Fourteen percent reported changing the seven letters into seven words that started with the corresponding target letter, but instead of creating a meaningful sentence, they then repeated these words subvocally. Twenty-seven percent reported changing the letters into a smaller number of words or nonsense words by adding vowels between the target letters. For example, the sequence $\mathrm{Y}, \mathrm{R}, \mathrm{J}, \mathrm{S}, \mathrm{L}, \mathrm{H}, \mathrm{Z}$ might be converted to two nonsense words such as YARJ, SALHAZ. These two words would then be repeated subvocally. The sequence B, C, D, $\mathrm{G}, \mathrm{P}, \mathrm{V}, \mathrm{T}$ might be converted to BOC, DIG, PRIVATE, which would then be repeated. Because they realized that the target letters were all consonants, these participants reported that they were able to avoid writing down vowels at recall.

Clearly, such a strategy would remove the phonological similarity effect because the participant would be unlikely to generate phonologically similar words. The use of this strategy and the semantic strategy fits well with what was predicted by Salamé and Baddeley (1986) when they were attempting to explain why the effects of irrelevant speech and phonological similarity tended to be additive at short list lengths and to interact at longer list lengths. Experiment 2 provides direct evidence that when lists contain seven items per sequence, some participants will adopt a strategy that negates the effects of variables such as phonological similarity and irrelevant speech. These results are also consistent with Baddeley's (1966) finding that with long sequences, serial recall is affected by semantic rather than phonological similarity between list items.

The relatively good performance of the semantic group might bring into question whether or not a phonological strategy is an effective means of retaining verbal sequences in short-term memory. It must be borne in mind, however, that the experimental manipulations that were employed in this experiment (phonologically similar lists, exposure to irrelevant speech, the use of supra-span lists) are specifically designed to put the phonologicalloop under extreme stress. Nevertheless, it is surprising that the semantic group performed so much better than the phonological group, and the effectiveness of semantic strategies in serial recall is well worth further investigation in the future.

\section{GENERAL DISCUSSION}

The results of both Experiments 1 and 2 have produced results that conflict with the predictions derived from Neath's (2000) feature model. Experiment 1 revealed additive effects of irrelevant speech and articulatory suppression with auditory presentation of list items. Neath claimed that the only way that such a result could occur would be if the irrelevant speech masked the presentation of the target items. In Experiment 1, however, similar results were obtained even when the irrelevant speech was presented after the target items. In Experiment 2, there were additive effects of irrelevant speech and phonological similarity with visual presentation as long as participants were asked to use phonological coding strategies. The feature model, however, predicts that irrelevant speech should abolish the phonological similarity effect with visual presentation when participants are using phonological encoding. ${ }^{3}$ Overall, therefore, these results cast doubt on Neath's (p. 419) claim that the feature model "correctly predicts the interactions between irrelevant speech and other factors long considered important in the study of immediate memory."

The basic problem is Neath's (2000) claim that irrelevant speech and articulatory suppression have broadly similar effects on serial recall. Hence it incorrectly predicts no combined effect of irrelevant speech and articulatory suppression in Experiment 1, and incorrectly predicts an interaction between phonological similarity and irrelevant speech in the phonological group in Experiment 2. The results, however, show that irrelevant speech can behave quite differently from articulatory suppression. For example, in the phonological group in Experiment 2, there were 
significant effects of phonological similarity on visually presented sequences in the presence of irrelevant speech. In the presence of articulatory suppression, however, it is well known that the effect of phonological similarity on visually presented sequences is abolished (e.g., Baddeley et al., 1984; Longoni et al., 1993; Murray, 1967, 1968).

Conversely, additive effects of irrelevant speech and articulatory suppression with auditory presentation of target items (Experiment 1) and additive effects of irrelevant speech and phonological similarity (Experiment 2) with visual presentation of target items follow exactly the predictions of the phonological loop model (e.g., Baddeley, 2000). The phonological loop model predicts effects of irrelevant speech in the presence of articulatory suppression as long as target materials are presented auditorily (Experiment 1). This is because articulatory suppression will not prevent auditorily presented material from entering the phonologicalloop, where it will become subject to interference from irrelevant speech. There can be phonological similarity effects on the serial recall of visually presented items even in the presence of irrelevant speech (Experiment 2) because irrelevant speech affects the storage of verbal materials within the phonological store but does not prevent access of visually presented materials to the phonologicalstore, according to the phonologicalloop model. The demonstration that phonologicalsimilarity effects are removed with semantic encoding instructions is also consistent with claims made by Salamé and Baddeley (1986) and Baddeley (2000). This finding provides support for Baddeley's (2000) suggestion that some previous studies (Colle \& Welsh, 1976; Surprenant et al., 1999) failed to obtain phonological similarity effects in the presence of irrelevant speech because some participants abandoned phonological encoding strategies as list length increased. Consequently, it appears that the phonological loop model provides a superior account of the irrelevant speech effect than does the feature model. ${ }^{4}$

The phonological loop model can also offer a possible explanation of why there are additive effects of irrelevant speech and phonological similarity with auditory presentation at list lengths in which nonsignificant effects of phonological similarity are observed with visual presentation (Surprenant et al., 1999). Because phonological presentation automatically registers target items in the phonological store, it seems reasonable to conclude that, relative to visual presentation, it will increase the probability that participants will employ phonological coding. Such an account could be tested by repeating Surprenant et al.'s (1999) study and asking participants to outline the strategies that they used. We predict that phonological strategies would be reported significantly more frequently with auditory presentation than with visual presentation at the sequence length that Surprenant et al. (1999) used.

One of the most attractive aspects of Neath's (2000) feature model is the fact that it can be implemented as a computational model. The model therefore contains an explicit account of the way in which serial order is retained. Performing simulations makes it possible to make precise predictions as to how the feature model will behave in the presence of one or more of the variables that are known to influence serial recall performance. It is also possible to predict the shape that the serial position curve will take. Computational models of the phonological loop have been developed in recent years (Burgess \& Hitch, 1999; Henson, 1998; Page \& Norris, 1998), but none of them have as yet attempted to explain the irrelevant speech effect. Our results suggest that the phonological loop model provides an appropriate framework for understanding a number of aspects of the irrelevant speech effect, but it is clear that a lot of important theoretical work remains to be done.

\section{REFERENCES}

BADDELEY, A. D. (1966). Short-term memory for word sequences as a function of acoustic, semantic and formal similarity. Quarterly Journal of Experimental Psychology, 18, 362-365.

BADDELEY, A. D. (1968). How does acoustic similarity influence shortterm memory? Quarterly Journal of Experimental Psychology, 20, 249-264.

BADDELEY, A. D. (1986). Working memory. Oxford: Oxford University Press, Clarendon Press.

Baddeley, A. D. (1990). Human memory: Theory and practice. London: Erlbaum.

BADDELEY, A. D. (1992). Is working memory working? Quarterly Journal of Experimental Psychology, 44A, 1-31.

BADDELey, A. D. (2000). The phonological loop and the irrelevant speech effect: Some comments on Neath (2000). Psychonomic Bulletin \& Review, 7, 544-549.

Baddeley, A. D., Lewis, V., \& VAllar, G. (1984). Exploring the articulatory loop. Quarterly Journal of Experimental Psychology, 36A, 233-252.

BRIDges, A. M., \& Jones, D. M. (1996). Word dose in the disruption of serial recall by irrelevant speech: Phonological confusion or changing state? Quarterly Journal of Experimental Psychology, 49A, 919-939.

Burgess, N., \& Hitch, G. J. (1999). Memory for serial order: A network model of the articulatory loop. Journal of Memory \& Language, $\mathbf{3 1}$, 429-460.

Colle, H. A., \& Welsh, A. (1976). Acoustic making in primary memory. Journal of Verbal Learning \& Verbal Behavior, 15, 17-32.

Conrad, R., \& Hull, A. J. (1964). Information, acoustic confusion and memory span. British Journal of Psychology, 55, 429-432.

Crowder, R. G. (1967). Prefix effects in immediate memory. Canadian Journal of Psychology, 21, 450-461.

Crowder, R. G. (1978). Mechanisms of backward masking in the stimulus suffix effect. Psychological Review, 85, 502-504.

Crowder, R. G., \& Morton, J. (1969). Pre-categorical acoustic storage (PAS). Perception \& Psychophysics, 5, 365-373.

Ellermeier, W., \& Zimmer, K. (1997). Individual differences in susceptibility to the irrelevant speech effect. Journal of the Acoustical Society of America, 102, 2191-2199.

Gathercole, S. E., \& Baddeley, A. D. (1993). Working memory and language. Hove, U.K.: Erlbaum.

HANLEY, J. R. (1997). Does articulatory suppression remove the irrelevant speech effect? Memory, 5, 423-431.

Hanley, J. R., \& Broadbent, C. (1987). The effects of unattended speech on serial recall following auditory presentation. British Journal of Psychology, 78, 287-297.

Henson, R. N. A. (1998). Short-term memory for serial order: The startend model. Cognitive Psychology, 36, 73-117.

Jones, D. M. (1994). Disruption of memory for lip read lists by irrelevant speech: Further support for the changing state hypothesis. Quarterly Journal of Experimental Psychology, 47A, 143-160.

Jones, D. M., \& MACKEN, W. J. (1993). Irrelevant tones produce an irrelevant speech effect: Implications for phonological coding in working memory. Journal of Experimental Psychology: Learning, Memory, \& Cognition, 19, 369-381.

Jones, D. M., \& Macken, W. J. (1995). Phonological similarity in the irrelevant speech effect: Within or between stream similarity. Journal 
of Experimental Psychology: Learning, Memory, \& Cognition, 21, 103-115.

Jones, D. M., Madden, C. A., \& Miles, C. (1992). Privileged access by irrelevant speech to short-term memory. Quarterly Journal of Experimental Psychology, 44A, 645-659.

Larsen, J. D., Baddeley, A. D., \& Andrade, J. D. (2000). Phonological similarity and irrelevant speech: Implications for models of shortterm verbal memory. Memory, 8, 145-158.

Longoni, A. M., Richardson, J. T. E., \& Aiello, A. (1993). Articulatory rehearsal and phonological storage in working memory. Memory \& Cognition, 21, 11-22.

Miles, C., Jones, D. M., \& Madden, C. A. (1991). Locus of the irrelevant speech effect in short-term memory. Journal of Experimental Psychology: Learning, Memory, \& Cognition, 17, 578-584.

Morton, J., Crowder, R. G., \& Prussin, H. (1971). Experiments with the stimulus suffix effect. Journal of Experimental Psychology, 91, 169-190.

Murray, D. J. (1967). The role of speech responses in short-term memory. Canadian Journal of Psychology, 21, 263-276.

Murray, D. J. (1968). Articulation and acoustic confusability. Journal of Experimental Psychology, 22, 679-684.

NAIRNE, J. S. (1990). A feature model of immediate memory. Memory \& Cognition, 18, 251-269.

NeAth, I. (2000). Modeling the effects of irrelevant speech on memory. Psychonomic Bulletin \& Review, 7, 403-423.

Neath, I., Farley, L. A., \& Surprenant, A. M. (in press). Directly assessing the relationship between irrelevant speech and articulatory suppression. Quarterly Journal of Experimental Psychology.

Neath, I., Surprenant, A. M., \& LeCompte, D. C. (1998). Irrelevant speech eliminates the word length effect. Memory \& Cognition, 26, 343-354.

Page, M. P. A., \& Norris, D. (1998). The primacy model: A new model of immediate serial recall. Psychological Review, 105, 761-781.

Salamé, P., \& BAdDEley, A. D. (1982). Disruption of short-term memory by irrelevant speech: Implications for the structure of working memory. Journal of Verbal Learning \& Verbal Behavior, 21, 150-164.

Salamé, P., \& BAdDEley, A. [D.] (1986). Phonologicalfactors in STM: Similarity and the unattended speech effect. Bulletin of the Psychonomic Society, 24, 263-265.

Surprenant, A. M., LeCompte, D. C., \& Neath, I. (2000). Manipulations of irrelevant information: Suffix effects with articulatory sup- pression and irrelevant speech. Quarterly Journal of Experimental Psychology, 53A, 325-348.

Surprenant, A. M., Neath, I., \& Lecompte, D. C. (1999). Irrelevant speech, phonological similarity, and presentation modality. Memory, 7, 405-420.

Watkins, M. J., \& Todres, A. K. (1980). Suffix effects manifest and concealed: Further evidence for a 20-second echo. Journal of Verbal Learning \& Verbal Behavior, 19, 46-53.

\section{NOTES}

1. The findings of Jones and Macken (1995) have sometimes been interpreted (e.g., Larsen et al., 2000) as being similar to those of Colle and Welsh (1976). In fact, Jones and Macken (Experiment 4) reported significant main effects of phonological similarity and irrelevant speech together with a nonsignificant interaction between these variables.

2 . Because articulatory suppression reduces recall more substantially than irrelevant speech, the value of a separate parameter labeled "attention" is also reduced in Neath's (2000) simulations of the effects of articulatory suppression.

3 . The version of the feature model that was implemented by Neath (2000; see p. 420) assumed that "the role of phonological information is constant." However Nairne's (1990) original model included a parameter designed to weight particular feature comparisons (see Neath, Farley, $\&$ Surprenant, in press). If one were to use this parameter to weight phonological features more heavily under phonological than under semantic coding instructions, then it might be possible that the feature model could simulate the performance of the phonological group in Figure 3 .

4. Nevertheless, there are some remaining weaknesses associated with the phonological loop model's explanation of the irrelevant speech effect. There is no account of the way in which serial order is represented within the model, and the fact that there is no additional effect of irrelevant speech when the speech and the targets are phonemically similar requires explanation. See Baddeley (2000) for a recent discussion of these issues.
(Manuscript received November 30, 2001; accepted for publication February 25, 2002.) 